\title{
Germanica
}

les arts dans les pays germanophones au $\mathrm{XX}^{\mathrm{e}}$ siècle

\section{Mythos und Erinnerung. Christoph Ransmayr: Die letzte Welt}

Myth and Memory in Christophe Ransmayr's Die letzte Welt

Mythe et mémoire. Christoph Ransmayr : Le dernier des mondes

\section{Rainer Godel}

\section{OpenEdition}

\section{Journals}

Édition électronique

URL : http://journals.openedition.org/germanica/827

DOI : $10.4000 /$ germanica. 827

ISSN : 2107-0784

\section{Éditeur}

Université de Lille

\section{Édition imprimée}

Date de publication : 30 décembre 2009

Pagination : 87-106

ISBN : 978-2-913857-24-7

ISSN : 0984-2632

\section{Référence électronique}

Rainer Godel, « Mythos und Erinnerung. Christoph Ransmayr: Die letzte Welt », Germanica [Online], 45| 2009, Online erschienen am: 01 Dezember 2011, abgerufen am 06 Oktober 2020. URL : http:// journals.openedition.org/germanica/827 ; DOI : https://doi.org/10.4000/germanica.827

Ce document a été généré automatiquement le 6 octobre 2020.

(c) Tous droits réservés 


\title{
Mythos und Erinnerung. Christoph Ransmayr: Die letzte Welt
}

\author{
Myth and Memory in Christophe Ransmayr's Die letzte Welt \\ Mythe et mémoire. Christoph Ransmayr : Le dernier des mondes
}

\section{Rainer Godel}

\section{I.}

1 In den vergangenen 30 Jahren hat einer der Grundtexte abendländischer Mythologie wieder verstärktes Interesse gefunden: Ovids Metamorphoses. ${ }^{1}$ Auch Christoph Ransmayrs viel besprochener Roman Die letzte Welt (1988) macht Ovids Verwandlungsmythen zum Material eines Spiels, das die Metamorphosen weiter- und umerzählt. Mythen bilden, so hat Thomas Anz ausgeführt, bei Ransmayr das Muster eines postmodern-offenen, autor- und ursprungslosen Textes. ${ }^{2}$ Damit entspreche der Roman Jacques Derridas kritischer Rezeption von Claude Lévi-Strauss' Mythenkonzept. Ransmayr erzähle „die Lebensgeschichte des Publius Ovidius Naso und die Überlieferungsgeschichte seiner Metamorphosen so um, daß sie postmodernen Postulaten [...] entsprechen. “ ${ }^{\text {I }}$ In der "mythopoetischen“ Fiktion manifestiere sich die Vorstellung, dass jeder Hörer das, was erzählt wird, durch seine Vorstellungen, Wünsche und Fähigkeiten mitpräge.

2 Doch ist diese „Arbeit am Mythos“" auch auf Kritik gestoßen. Christian Schärf glaubt in Die letzte Welt eine „Zauberkiste der Motivzitate“ zu erkennen, „,ie immer nur wieder auf sich selbst zurückverweisen“; Kurt Bartsch kontrastiert den durch Ransmayr ,wiederbelebten' Mythos mit der Aufklärung; ${ }^{6}$ Altphilologen konstatieren angesichts der Unterschiede zwischen Ovid und Ransmayr nicht selten ein Qualitätsgefälle zu Ungunsten des Gegenwartsautors. ${ }^{7}$ Peter Bachmann spricht in einem der ersten Beiträge, die Ransmayrs Roman mit der Problematik des Mythos in Zusammenhang bringen, von „prätentiösem Kunsthandwerk“ “. Der Text gefalle ihm daher nicht. Nun sei dahingestellt, ob "Gefallen“ die Qualität eines literaturwissenschaftlichen Arguments hat. Unstrittig aber dürfte sein, dass erst die Kontextualisierung von 
Ransmayrs „Arbeit am Mythos“ es ermöglicht, der spezifischen Problemlage gerechter zu werden. Mein Anliegen ist es zu verdeutlichen, welche Funktion die Transformation des Mythos im literarischen Wirkungszusammenhang bei Ransmayr übernimmt. Die Motivzitate, das Aufrufen und die Variation des Mythos machen - so meine These eine poetologisch unterlegte Differenz deutlich, die die Verstehensleistung des Lesers durch Distanzierung und Reflexionsanreiz aktiviert. Dabei liegt das punctum saliens der Funktion der „Arbeit am Mythos“ nicht in einer vorgängigen postmodernen oder neostrukturalistischen Poetik, sondern in Ransmayrs Poetik der Erinnerung. ${ }^{9}$

„Wenn man spricht, erzählt oder schreibt - von all dem, was in der Erinnerung und Sprache der Menschen zu Geschichten geworden ist -, dann streift man zumindest inhaltlich und manchmal vielleicht auch formal unwillkürlich an alle Bemühungen, die sich je mit ähnlichen Themen beschäftigt haben. ${ }^{10}$ So deutet Christoph Ransmayr in den Geständnissen eines Touristen (2004) sein literarisches Verfahren, mit zu Geschichten gewordenen Mythen literarisch neu umzugehen. Er beschreibt die Aneignung des Vergangenen als in inhaltlicher wie formaler Hinsicht, leichte Berührung', nicht als ,dichte Beschreibung, die ein teilnehmendes Eindringen in das kulturelle Erbe ermöglichte. Er zielt darauf, mit seiner literarischen Umformung des Mythos eine nicht-traditionale Neuerzählung unter den Bedingungen der Gegenwart zu schaffen, die nicht Vergangenes referiert, sondern Geschichten und Mythen neu und umerzählt.

Dabei bilden Ransmayrs Texte nicht Realität ab. Denn Erinnerung formt die Repräsentation von Mythos und Geschichte im Erzählen. In Die dritte Luft, seiner Eröffnungsrede $\mathrm{zu}$ den Salzburger Festspielen 1997, erzählt Ransmayr von einer öffentlichen Bühne im Süden Irlands, einer auf einer Klippe liegenden Plattform, die genutzt wird, um Geschichten zu erzählen, zu spielen, zu feiern. Im Moment des Spielens oder Erzählens auf dieser Bühne lassen die Mitspieler „eine ganze Welt“ hinter sich und treten „,in eine Melodie ein, in eine Ballade, einen Applaus oder ein Gelächter, in dem [ihnen ihr] Leben plötzlich noch einmal neu und anders erschien." ${ }^{11}$ Die erzählte Erinnerung verändert als Fiktion die Wahrnehmung der Gegenwart. Denn in der erzählten Geschichte befindet sich der Erzähler - so beschreibt der Autor es in Die Erfindung der Welt - in der „Mitte der Welt“, in der alle Welt noch einmal erfunden wird. ${ }^{12}$ Erinnerung ist Fiktion, da sie nicht ,Fakten' wiedergibt, sondern sie ,nur' erzählt. Die „bloße[n] Geschichten“ dienen dazu, das „Repertoire, das Gedächtnis der Plattform“ zu erneuern. ${ }^{13}$ Vergangenes wird in diesen Geschichten nicht mimetisch dargestellt, sondern für die Gegenwart re-konstruiert. Die Gegenwart verbindet dabei die erinnerte, aber auch die mythologisch verarbeitete Vergangenheit in einer Parabel, nicht in einer Gerade mit der Zukunft. „Was aber könne in einer Geschichte, auf einer Bühne, denn Größeres getan werden, als daran zu erinnern, was die Menschen sich selbst und einer dem anderen angetan hätten, daran zu erinnern, wozu diese Menschen in ihrem Glück und in ihrem Elend fähig seien und was ihnen also auch in Zukunft noch winken oder drohen werde?"14 Erinnert wird also an Möglichkeiten, wie die Vergangenheit gewesen sein und wie die Zukunft werden könnte. Diese Möglichkeiten konkretisieren sich im „Kopf“, in der „Vorstellungskraft des Erzählers und seiner Zuhörer“: „Erst dort wird die Welt schließlich vollständig, nur im Erzählraum liegen Mögliches, zumindest Plausibles - und Notwendiges, Tatsächliches, kurz: alles, was der Fall ist - bloß durch hauchdünne, oszillierende Membrane getrennt nebeneinander." 15 Die imaginative Fähigkeit des Lesers erzeugt im Lesen und Zuhören das, was Hans 
Blumenberg als Funktion des Mythos bestimmt hat: „Welt zu haben“. Dies ist nach Blumenberg „immer das Resultat einer Kunst“, der „Arbeit am Mythos“. ${ }^{16}$

Die Wirklichkeit, die im Erzählen wie im Mythos erzeugt wird, verbindet Vergangenheit und Zukunft $\mathrm{zu}$ einem erzählten Kontinuum, das die jeweilige Aktualisierung von Erinnerung zum Gegenstand hat. Nicht die realhistorische Vergangenheit wird in Mythos und Erzählung erinnert und nicht sie könnte nachgebildet werden. Vielmehr wird eine erzählte und daher fingierte Vergangenheit zum Ausgangspunkt des Weiter-Erzählens. Die fiktionale Situation, die „steinerne Bühne“ bei Ransmayr, erscheint als „Angelpunkt“, „als der einzige feste Ort, um den sich die Wirklichkeit und das ganze Land zu drehen begann. ${ }^{\text {"17 }}$ Erzählen bildet die reale Vergangenheit nicht $a b$, es gestaltet sie in der individuellen Erinnerung neu. Die narrative Variation erzeugt Geschichten, die bildstarke Erinnerungen konstruieren. Erinnerung muss erzählt werden. Den Unterschied von Geschichte und Erinnerung markiert ein spezifisch narrativer Akt, der auf individueller memoria beruht, der Perzeptions- und Selektionsprozesse voraussetzt und diese erzählerisch individuell gestaltet. Entgegen Jean-François Lyotards Diagnose vom Verlust der großen Erzählungen ${ }^{18}$ ist in Ransmayrs Autorpoetik die Sinn gebende Metaerzählung immer gegenwärtig: „auf Glaisín Álainn wurde weder am Torffeuer noch auf der Plattform jemals vergessen, zu welchem Stück alle die Geschichten, Tänze und Lieder gehörten, [...]. ${ }^{{ }^{19}}$ Die Situation des Erzählens selbst wird Gegenstand der erzählten Erinnerung. Die Bühne ist „längst selbst zur Geschichte geworden, zur Erinnerung und Provokation der Phantasie." ${ }^{\prime 20}$

6 Auf der Grundlage dieser narrativen Poetik verbindet Ransmayr Erinnerung und Zeit überschreitende Utopie. Hierzu nutzt er die Funktionsweise des Mythos. Es wird eine Zeit fingiert, die sich von der Realhistorie ablöst und die den Rezipienten dazu anleitet, Leerstellen selbstständig auszufüllen. In Ransmayrs Texten geht es nicht um den narrativen Charakter von Geschichte, es geht, folgt man seiner Poetik, um die Bedingungen der Konstituierung von zeitlos-erinnerten, individuellen Geschichten. ${ }^{21}$ In diesem Sinne ist auf poetologischer Ebene die Anverwandlung und Form von Mythen in Ransmayrs Texten folgerichtig. Nimmt man Ransmayrs poetologische Selbstauskunft ernst, so handelt es sich bei Literatur um ein Transformationsprojekt, das den Bedingungen literarischer Intertextualität und ,mythologischer' Erinnerung unterliegt.

Die spezifische Verbindung von Zeiten übergreifender Wirksamkeit und je zeitbedingt geprägter Transformation macht den Mythos (und das Erzählen bei Ransmayr) aus. Blumenberg akzentuiert in Arbeit am Mythos, Cassirer und Claude Lévi-Strauss kritisierend, genau jene beiden Aspekte, die auch für die Poetik Ransmayrs distinkt werden. Nicht dadurch, dass ein bestimmter Inhalt in zeitliche Ferne gerückt und in die Tiefe der Vergangenheit zurückgelegt wird, erhält er nach Blumenberg mythische Qualität, sondern die Arbeit des Mythos gründe darin, dass er zeitlich stabil sei. Doch dürfe man temporale Resistenz und Zeitlosigkeit nicht verwechseln. ${ }^{22}$ Lévi-Strauss' These, der innere Wert des Mythos stamme daher, dass die zu Grunde liegenden Ereignisse eine "Dauerstruktur" bildeten, die sich „gleichzeitig auf Vergangenheit, Gegenwart und Zukunft" beziehe, ${ }^{23}$ geht für Blumenberg $\mathrm{zu}$ weit. Erst die Zeitbestimmtheit des Früher und Später macht für Blumenberg die Unterschiede der jeweiligen Aktualisierungen der Mythen aus. ${ }^{24}$ Die je temporale Ordnungsform sei entscheidend für die Konstitution eines Mythos. ${ }^{25}$ Dieser manifestiert sich indes, wie Stephanie Wodianka gezeigt hat, nicht als „Erinnerungsreferenz“, als Abbild einer 
,faktischen' Erinnerung, sondern als „Erinnerungsperformanz“, die den konstruktiven Charakter der Erinnerung unter je spezifischen Gegebenheiten fiktional gestaltet. ${ }^{26}$ „Nur wenn man den Mythos als Distanz zu dem versteht, was er schon hinter sich gelassen hat [...]", so resümiert Blumenberg, ,kann man den Spielraum der Imagination als das Prinzip seiner immanenten Logik begreifen, aus der die Grundformen der Umständlichkeit und Umwegigkeit, der Wiederholung und Integration, der Antithese und Parallele hervorgehen." ${ }^{27}$

\section{II.}

Wenn zutrifft, dass Mythen immer schon in Rezeption übergegangen sind und dass die Arbeit am Mythos im Bereich der Fiktion, im „Spielraum der Imagination“ stattfindet, ${ }^{28}$ und wenn zugleich Ransmayrs Verständnis von Erzählen genau jene Elemente der Konstruktivität der Erinnerung betont, die die Arbeit am Mythos prägen, kann es dann verwundern, dass auch seine Arbeiten zu Ovid sich schnell von einer Translation zu einer Transformation entwickelten? Hans Magnus Enzensberger hatte Ransmayr gebeten, Ovids Metamorphosen neu zu übersetzen. ${ }^{29}$ Schon das erste Probestück, die knappe Erzählung Das Labyrinth, ${ }^{30}$ formt die Vorlage zu neuen Perspektiven um. Der Kern des Mythos bleibt erhalten: Minos beschließt, Minotaurus in einem Labyrinth gefangen zu setzen, das Daedalus, der nach Kreta verbannte Baumeister, errichtet. Ransmayrs Erzählung fokussiert den Moment, in dem Minos Daedalus seinen Befehl erteilt. Die Parallele zwischen Ovid und Ransmayr liegt also in der zu Grunde liegenden Machtstruktur. Ein exilierter Baumeister wird vom Herrscher seines Gastlandes zum Errichten eines Labyrinths gezwungen. Und das Unternehmen Daedalus' gelingt bei Ovid gut, der Baumeister findet den Weg zur Schwelle kaum mehr zurück: „vixque ipse reverti / ad limen potuit" ${ }^{31}$ Doch während bei Ovid der Baumeister bloß droht, die Orientierung zu verlieren, hat er in Ransmayrs Erzählung einen eigenen Standpunkt bereits eingebüßt. Daedalus hat keine Sprache mehr, für Ransmayr die Ausdrucksform von Geschichten. Er spricht nicht selbst in Ransmayrs Erzählung. Der Satz, der offenlegt, dass auch Minos Machtstrukturen unterliegt, denen er nicht entkommen kann, wird nicht von Daedalus, sondern parodistisch von Minos selbst gesprochen, nachdem er vorher aposiopetisch ausgelassen worden war: „Da trat der Kreter so dicht an ihn heran, daß er seinen Atem roch, und wiederholte nun selbst und äffte dabei den Tonfall des Untertanen nach: Herr, du sprichst von deinem eigenen Palast." ${ }^{32}$ Das Labyrinth kann als früher Versuch gelten, die Funktion des Mythos zu nutzen, um ihn zu transformieren und mit anderen Propositionen zu verbinden..$^{33}$

9 Analog gilt für Ransmayrs Roman Die letzte Welt: Der Roman transformiert die alten Mythen unter den Bedingungen von Ransmayrs Poetik der Erinnerung. Noch stärker als im Labyrinth tritt dabei der Charakter der Erinnerungsarbeit am Mythos in den Vordergrund. Die letzte Welt ist damit kein Teil des „Mythologems“, des ritualisierten Textbestandes, ${ }^{34}$ sondern sie arbeitet am Mythos, indem sie den Textbestand der mythologischen Erinnerung überschreitet. Damit amalgamiert der Roman Formen der Erinnerung. ${ }^{35}$ Ransmayrs Text bezieht sich in vielfacher Weise auf Ovids Metamorphoseon libri. ${ }^{36}$ Analysiert man die narrative Struktur des Romans, so werden indes Differenzen sichtbar, die über die bloße Aufnahme von mythologischen Motiven hinausgehen. Die Adäquanz an Ovid ist für den literarischen Text der Gegenwart nicht erforderlich, zumal Verwandlung als Prinzip des Umgangs mit Mythen bei Ovid selbst 
poetologisch gerechtfertigt wird: „Ante mare et terras et, quod tegit omnia, caelum / unus erat toto naturae vultus in orbe, / quem dixere Chaos, [...]. ${ }^{{ }^{37}}$ Das Chaos des Anfangs der Welt erfordert zwingend Veränderung und Verwandlung. ${ }^{38}$ Es zieht Metamorphosen nach sich.

Der Mythos gewinnt bei Ransmayr eine zusätzliche Funktion, die von der poetologischen Konzeption geleitet wird. Die literarische Form, innerhalb derer Mythen in Die letzte Welt transformiert werden, erzeugt eine neuartige Komplexität, die nicht mehr die Komplexität des Mythos aus der Sicht des „Verbrauchers“ (im Sinne Roland Barthes') ist. Denn das Selbstverständlich-Komplexe der antiken Mythologie, in der der Mythos gleichzeitig als unentwirrbar und als wahr gelesen werden konnte, wird bei Ransmayr durch eine poetologische Funktion ersetzt, die die Unentwirrbarkeit und Wahrheit der Erinnerung behauptet. ${ }^{39}$ Die Poetologie der Erinnerung ermöglicht es, Vergangenheit und Zukunft in der Erzählung zu verbinden. Diese Umformung nimmt ernst, dass Mythen nicht eine definitive Form haben, sondern in einer Vielzahl von mündlichen und schriftlichen Varianten vorliegen können.

Cotta macht sich in Die letzte Welt in Tomi auf die Suche nach dem dort exilierten Autor Naso und nach einer Abschrift von dessen Metamorphoses. Doch Cotta findet den Dichter nicht. Stattdessen findet er Erinnerungen: Er nimmt die erzählte, geknüpfte, in Stein gemeißelte Erinnerung der Bewohner wahr, die von Naso berichten oder Geschichten erzählen, die sie Naso zuschreiben. Cottas Versuch, die Metamorphoses zu rekonstruieren, wird gespeist aus den Erzählungen Echos, aus den Teppichen Arachnes, aus den Steinmälern des Pythagoras. Jede Figur gestaltet ihre individuelle Erinnerung an Naso und an sein verschollenes Buch. Die Metamorphoses finden sich nicht in Form eines Buches, sondern in den Figuren selbst, in ihren Erzählungen, Filmen und Verwandlungen. Die Geschichten Nasos haben sich in eine Welt verwandelt. Der Mythos ist zur erzählten Realität geworden. Doch können weder Cotta noch die Leser des Buches entscheiden, ob es sich um eigene Phantasie der Figuren oder aber um eine ,historische' Relation der Erzählungen Nasos handelt. „Hatte Naso jedem seiner Zuhörer ein anderes Fenster in das Reich seiner Vorstellungen geöffnet, jedem nur die Geschichten erzählt, die er hören wollte oder zu hören imstande war?" ${ }^{40}$ So stellt sich für Cotta wie für den Rezipienten die Frage: Wie verlässlich ist die Rekonstruktion von Erinnerung?

Während also das mythologische Programm der Verwandlung und Veränderung in Ransmayrs Roman überdauert, haben die einzelnen Mytheme ihre Funktion zunächst anscheinend verloren. Auf der diegetischen Ebene des Textes werden sie, anders als bei Ovid, nicht direkt mit einer sichtbaren Deutung verbunden. „nomenque erit indelebile nostrum “:41 Das programmatische Dichternachwort bildet den Schluss des letzten Buchs der Metamorphosen. Hier wird die Unzerstörbarkeit, die Dauerhaftigkeit und Zeitlosigkeit der Literatur jenseits aller Verwandlungen angekündigt. Für den Leser, der die Bücher von den Verwandlungen in Händen hält, wird diese Vorausschau unmittelbar einsichtig. Ransmayr übernimmt zwar große Teile dieses Nachworts in seinen Roman, doch ist dessen Wortlaut im Roman in Trachila, einem kaum zugänglichen Ort, auf Steinmalen eingeschrieben. Dort ist die in Stein gemeißelte „Botschaft auf Basalt und Granit“" unter Schnecken versteckt, die erst getötet und beseitigt werden müssen, bevor eine lesbare Schrift erscheint. ${ }^{42}$ Was bei Ovid als transparente Rede des Autors in Ich-Form erscheint, ist in Ransmayrs Mythos- 
Transformation zum Geheimnis geworden, dessen sich der Protagonist und mit ihm der Leser bemächtigt.

Ransmayr hat gegenüber Ovids Fassung Akzente auch durch Auslassung anders gesetzt. Er erweitert die auf die Zukunft gerichtete Prophezeiung des zitierten Verses, indem er die politische Präzisierung, die den Gedanken der Dauerhaftigkeit des literarischen Ruhms zeitlich und räumlich einschränkt, auslässt. Während sich bei Ovid an das wörtlich von Ransmayr übernommene Zitat „nomenque erit indelebile nostrum“ - bei Ransmayr: „Mein Name wird unzerstörbar sein“" ${ }^{43}$ - die Konkretisierung anschließt: "quaque patet domitis Romana potentia terris“, ${ }^{44}$ lässt Ransmayr dies aus. Bei Ransmayr wird die zukünftige Gültigkeit der Prognose nicht mehr durch eine zeitlichräumliche Konkretisierung eingeschränkt. Die Variation von Ovids zentralen programmatischen Passagen signalisiert bei Ransmayr eine Transformation des Mythos, die insbesondere die zeitliche Dimension betrifft. Dies wird umso deutlicher, zieht man Welt- und Menschenerschaffungsmythen - wohl die Urform mythologischer Erzählung - heran. Von Ovid übernimmt Ransmayr die Erzählung zweier Mythen, die vom (Wieder-)Anfang des Menschengeschlechts erzählen: Cotta erinnert sich in Die letzte Welt an Nasos öffentliche Rede, in der er vom Geschlecht der Ameisenmenschen aus Aegina erzählt, und Echo erzählt den Mythos von Deucalion und Pyrrha und der Schaffung des Menschen aus Steinen.

In Ovids Metamorphosen ist die Erzählung der Ameisenmenschen aus Aegina, der Myrmidonen, in ein Hilfeersuchen eingebettet. Der Athener Cephalus kommt nach Aegina, um von König Aecus Hilfe im Krieg gegen Minos zu erbitten. ${ }^{45}$ Cephalus stellt fest, dass viele derjenigen, die er bei einem früheren Besuch gesehen hatte, nun nicht mehr anzutreffen sind. Aecus erzählt daraufhin von der vernichtenden Pest und von der Neuschöpfung eines Menschengeschlechts aus Ameisen. Die Geschichte fokussiert das Leid des Aecus, der der Gewalt der Götter ausgeliefert ist. Durch den Zorn der Iuno bricht die verheerende Pest aus, der große Teile der Bevölkerung zum Opfer fallen, durch den Einfluss Jupiters entsteht ein neues Geschlecht. Aecus berichtet über eine abgeschlossene Verwandlung von Ameisen in Menschen: „mores, quos ante gerebant, nunc quoque habent ${ }^{\text {“. }}{ }^{46}$ Die Erzählung des Aecus erläutert also einen gegenwärtigen Zustand, indem er seine Genese darstellt. Mit dem Versprechen des Cephalus, Aecus die neuen Krieger zur Verfügung zu stellen, wird der Mythos der Erschaffung von Menschen von der Vergangenheit über die Gegenwart bis in die Zukunft vermittelt.

In Die letzte Welt berichtet der Erzähler, dass Naso in einer öffentlichen Rede vor dem voll besetzten Stadion in Rom die Geschichte der Pest auf Aegina erzählt hat. Aus der Erzählung eines vergangenen Ereignisses, die bei Ovid innerhalb der Fiktion Realität beansprucht - denn Aecus erzählt innerhalb der Metamorphosen eine ,wahre' Geschichte, die die Erzählgegenwart Aecus' erklärt - wird bei Ransmayr ein Gleichnis, das auf der intradiegetischen Ebene als Analogie verstanden werden soll. Nasos Rede weist analogisch auf die Zukunft: „Und was die Eiche der Ameisen für das Glück der Insel Aegina war, sagte Naso dann in den Strauß der Mikrophone und schloß seine Rede, das werde nun und in Zukunft dieses Bauwerk der Sümpfe, das Stadion Zu den Sieben Zufluchten, für das Glück Roms sein - ein Ort der Verwandlung und Wiedergeburt, ein steinerner Kessel, in dem aus Hunderttausenden Ausgelieferten, Untertanen und Hilflosen ein Volk gekocht werde, so wandelbar und zäh wie das neue Geschlecht von Aegina, so unbesiegbar.“" ${ }^{47}$ Mit diesem Schluss der Rede formuliert Naso die Hoffnung darauf, dass in der Zukunft sich ein Geschehen ereignen werde, welches 
seiner Deutung des erzählten Mythems entspricht. Zur Sprache kommt auf Erzählebene eine Hoffnung auf die Zukunft, die auf einer expliziten Kritik beherrschbarer und duldsamer Volksmassen fußt. ${ }^{48}$ Während also in der Erzählung des Aecus bei Ovid eine Erklärung für die Gegenwart geboten wird, die Elemente der Kritik an der Willkür der Götter und an den Machtansprüchen der irdischen Herrscher birgt, transformiert Ransmayr erzählerisch das Mythem zu einer erzählten Utopie in der Zukunft, die Verwandlungen hypothetisch durchspielt. ${ }^{49}$

In Bezug auf die Zeitstruktur lassen sich zum zweiten Schöpfungsmythos, der sowohl bei Ovid als auch bei Ransmayr thematisch wird, ähnliche Überlegungen anschließen. In beiden Texten wird von der großen Flut erzählt, an deren Ende Deucalion und Pyrrha, die einzigen Überlebenden, Steine hinter ihre Rücken werfen, die sich in ein neues Menschengeschlecht verwandeln. ${ }^{50}$ Der zentrale Unterschied liegt auch hier in der Zeitdimension: Ovid bietet mit der Erzählung von Deucalion und Pyrrha eine mythische Erklärung für die gegenwärtige Menschheit: „inde genus durum sumus experiensque laborum / et documenta damus, qua simus origine nati.“51 Indem das nach den Steinwürfen aus der Metamorphose entstandene Menschengeschlecht vom Erzähler als dasjenige der Erzählergegenwart identifiziert wird, situiert er den Prozess der Verwandlung als Zwischenstufe zwischen der göttlichen Prophezeiung und deren Erfüllung in der Text- und Lesergegenwart. ${ }^{52}$

Die mythische Erzählung hat auch bei Ransmayr ihren Ort in einer imaginierten Vergangenheit, doch gilt sie im Moment des Erzählens als Erinnerung an die Zukunft: „Aus einem Steinhagel, schrie Echo, werde nach der kommenden, allesvernichtenden Flut die neue Menschheit hervorgehen, - diese Zukunft habe ihr Naso an einem Wintertag aus dem Feuer gelesen, [...]." ${ }^{453}$ Aus dem Schöpfungsmythos, der bei Ovid die Erklärung für die Lesergegenwart in der Vergangenheit sucht, ist bei Ransmayr die Prognose der Zukunft auf Erzählebene geworden: „und dennoch traute Cotta seinen Ohren kaum, als sie ihm zuschrie, er werde nun die Geschichte des bevorstehenden Untergangs der Welt hören, eine Offenbarung der Zukunft. " ${ }^{44}$ Echo erzählt ihre Erinnerung an eine Erzählung Nasos, die „letzte Erzählung aus dem Buch der Steine“, ${ }^{55}$ die in eine zukünftige Transformation überführt werden wird. Ihre Erzählung verlegt die Erfüllung des Mythos in die Zukunft. Sie „beschrieb die künftige Flut so bestimmt wie eine Katastrophe der Vergangenheit." ${ }^{56}$ Die Gegenwart bleibt ausgespart. Die Zeitdifferenz von erinnerter Vergangenheit und prognostizierter Zukunft erzeugt hier eine zukunftsgewisse Alternative, die nur in der Nicht-Zeit der Fiktion zustande kommen kann. Am Ende steht eine neue, ,die eigentliche und wahre Menschheit“, die nach dem Niedergang des , an seiner wölfischen Gier, seiner Blödheit und Herrschsucht zugrundegegangenen Geschlechts hervorkriechen werde“, eine Menschheit „von mineralischer Härte, [...] ohne Gefühle, ohne eine Sprache der Liebe, aber auch ohne jede Regung des Hasses, des Mitgefühls und der Trauer ${ }^{4{ }^{57}}$ Echo berichtet hier aus ihrer Erinnerung die Prognose einer innerlich versteinerten Menschheit, die sich auf dramatische Weise den mineralisch-harten und unempfindlichen Formen des Steines angleicht. ${ }^{58}$

18 Setzt man die Neuerzählung des Deucalion und Pyrrha-Mythems in den Zusammenhang von Ransmayrs Poetologie, so wird deutlich, dass der erzählte Verlauf der Zeit bei Ransmayr von einer entscheidenden Leerstelle geprägt ist: von der Leerstelle der erzählten und rezipierten Gegenwart. Echos individuelle Erinnerung wird erzählt und eben keine mythisch oder apokalyptisch allgemeine. Cotta hatte von dieser 
Erzählung „,in den Reden und römischen Lesungen Nasos niemals gehört ““ ${ }^{59}$ Dass Cotta diese Erzählung nicht kennt, belegt indes nicht die „Schwundstufe des Mythischen“ in Rom, ${ }^{60}$ sondern repräsentiert die Individualität des Erinnerns gegen den kollektiven, für alle Zeit gültigen Mythos. Was hier erzählt wird, ist nicht mehr ein tradierter Mythos, der die Sintflut und ihre Folgen für die Gegenwart darstellt, es ist die individuelle Erinnerung Echos an eine Zukunftsprognose, die mit allen Merkmalen persönlicher emotionaler Betroffenheit der Erzählerin verbunden und in eine erzählerisch und motivisch abweichende Situation eingebettet ist. ${ }^{61}$ Eine solche parabolische Verbindung von Erinnerung und Utopie, von Vergangenheit und Zukunft, die nicht die Gegenwart erklärt, bildet das Strukturprinzip von Ransmayrs Letzter Welt. Durch Erzählungen von Figuren und über sie wird der Weg in ein Außerhalb-der-Zeit-Stehen vorgezeichnet. ${ }^{62}$

19 Ransmayr zielt also nicht darauf ab, aus der Transformation des alten einen neuen Mythos zu generieren, der in der Gegenwart des Rezipienten als Legitimierung eines Weltverständnisses genutzt werden könnte. Aber die Funktionsweise der Mythen geht in ihre Transformation ein. Die "Objektivität" des Mythos gründet sich nach Ernst Cassirer darin, „daß er nicht das Abbild eines gegebenen Daseins, sondern eine eigene typische Weise des Bildens selbst ist, in der das Bewußtsein aus der bloßen Rezeptivität des sinnlichen Eindrucks heraus- und ihr gegenübertritt." ${ }^{63}$ Auch Ransmayrs Text zeigt, wie aus der individuellen ,Erinnerung' heraus Bilder, Erzählungen, Fiktionen generiert werden, die Erwartungen, Hoffnungen und Befürchtungen für die Zukunft formulieren. Das verwandelnde Erzählen übernimmt die Funktion eines Mythos, nicht nur, indem der neue Mythos soziale Erfahrung objektiviert, sondern auch indem das Erzählte zur Keimzelle zukünftiger Erfahrung werden kann. ${ }^{64}$ Die Arbeit am Mythos geht in die Arbeit des Mythos unmittelbar über.

Dies trifft sowohl für das Erzählen selbst, in dem dieser Vorgang thematisch wird, als auch für die Text-Leser-Relation zu. Ransmayr macht Funktionen des Mythos sichtbar. Doch greift er nicht alle Funktionen in identischer Weise in bloßen Variationen auf. Er kontrastiert zwei mögliche Transformationen des Mythos im „Ovidischen Repertoire“, das den Roman beschließt. ${ }^{65}$ Ransmayr stellt in diesem „Repertoire“ in zwei Spalten die "Alte“ und die „Letzte Welt" gegenüber, Figuren aus Ovids Metamorphosen und aus Ransmayrs Die letzte Welt. Das „Repertoire“ dient dabei nicht bloß dazu, mythologische Beständigkeit und Veränderbarkeit ${ }^{66}$ gegenüberzustellen. Es manifestiert eine strukturelle Trennung, die die Möglichkeiten der Fiktion aufzeigt, von kollektiven Erinnerungen abzuweichen, individuelle Geschichten neu zu erzählen und gerade auf diese Weise zur Arbeit des Mythos beizutragen. Das „Repertoire“67 kontrastiert zwei fiktiv-imaginäre Erinnerungskonstruktionen. Insofern parodiert es Glossare aus historischen Romanen und aus Ovid-Ausgaben, welche durch erläuternde Kommentare Bedeutungen festschreiben und eben nicht die Vielfalt individueller Deutungsmöglichkeiten aufzeigen. Das „Repertoire“ in Die letzte Welt jedoch demonstriert die Vielfalt der Fiktion, die dennoch nicht dem Prinzip des Mythos widerspricht. ${ }^{68}$ Das „Ovidische Repertoire“ ist das „Gedächtnis“ des Mythos, eine Sammlung von potenziellen Fiktionen.

21 Schon in Die dritte Luft verwendet Ransmayr den Begriff des "Repertoires“. Hier kennzeichnet er damit die erzählte Wiederkehr der „Verschwundenen“, „Ausgewanderten“ und „Toten“, die an Winterabenden auf der Bühne in Irland durch „Geschichten und Lieder[n]“ geschieht. ${ }^{99}$ Das „Repertoire der Plattform“ wird im Erzählen erneuert, doch nicht, um durch das Erinnern einer historischen Realität näher 
zu kommen: „So viele Geschichten und Songs könnten doch von allen Dichtern und Sängern dieser Welt nicht erzählt und nicht gesungen werden, um alle [...] verborgenen Dramen und Tragödien dem Gedächtnis der Menschheit zu bewahren. " ${ }^{\text {"70 }} \mathrm{Im}$ Erzählen vollziehen sich Verwandlungen. Es „verwandle sich beispielsweise ein ganzes Meer in ein einziges Wort, in eine Melodie, und rausche aus diesem Wort wieder hervor. “" ${ }^{71}$ Ein "Repertoire“ verbindet also die individuelle Erinnerung mit einer symbolischen Deutung. In der Gegenüberstellung der beiden Welten wird dieser Charakter von Fiktion deutlich. Die „Bearbeitung“ Ovids erscheint als individuell und legitim. Gleichzeitig demonstriert sie nur eine der unendlichen Möglichkeiten der Mythenproduktion und -rezeption. ${ }^{22}$

In Ransmayrs Die letzte Welt bleibt trotz des „Repertoires“ die realhistorische Gegenwart auf Erzählebene unterbestimmt. Erzählt wird von einer anderen Historie, in der die Zeitenfolge des Erwartungshorizonts der Leser aufgehoben ist. Die Gültigkeit des antiken Mythos als Welterklärung ist suspendiert, doch tritt keine andere Welterklärung an seine Stelle. Der temporalen Stabilität des mythischen Kernbestandes steht in Ransmayrs Roman die Auflösung des zeitlichen Bezugsrahmens gegenüber. Die letzte Welt weicht von Geschichte wie von Geschichten ab. Der Roman spielt weder räumlich noch zeitlich im alten Rom und in seiner peripheren Kolonie Tomi. Die Geschichte ist in eine ahistorische, zeitlose, fiktive Zeit versetzt, die Elemente der realhistorischen Antike mit Elementen späterer Zeiten verbindet. ${ }^{73}$ Durch die Vermischung von historischen Figuren und modernen Requisiten entsteht ein eigener, keiner bestimmten außerliterarischen Wirklichkeit mehr zuzuordnender Zeit-Raum, in welchem sich Cotta von Anfang an befindet. ${ }^{74}$ Ransmayr bestätigt dies selbst: „Denn es ging in dieser Letzten Welt eben nicht! darum, mit Anachronismen im Sinne einer ziemlich trostlosen postmodernen Willkür bloß zu spielen, sondern durch ein Netz zeitlicher Bruchlinien eine Unzeit, Allzeit zu provozieren, einen narrativen, keinen historischen Raum und erst aus ihm heraus mit allen, der Deutlichkeit und Plausibilität dienenden Mitteln zu erzählen." ${ }^{\text {75 }}$ Gerade die Anachronismen der Letzten Welt konstituieren eine abweichende Zeitstruktur.

Cottas Schiffsreise nach Tomi, nach dem „Irgendwo“ ", ${ }^{76}$ nach dem nicht fixierbaren Ort, beginnt im April, doch setzen die Umstände der Reise den Protagonisten gleichsam außerhalb messbarer Zeit. Der den Tagesablauf des Menschen strukturierende Wechsel von Wach- und Schlafzustand ist aufgehoben. "Niemand schlief." scheinen räumlich-politische Bezugspunkte wie jahreszeitliche Gewohnheiten von Beginn der Erzählung an aufgehoben: „Aber Rom war in diesen Tagen ferner als sonst. Denn in Tomi hatte man sich von der Welt abgewandt, um das Ende eines zweijährigen Winters zu feiern. ${ }^{\text {" }}{ }^{78} \mathrm{Am}$ Ende des Romans erfährt Cotta dies als Durchdringung der Zeit: „Die Zeiten streiften ihre Namen ab, gingen ineinander über, durchdrangen einander." ${ }^{79}$ Von Beginn des Romans an ist Erinnerung als Differenz gezeichnet. Als Cotta in Tomi anlangt, stellt der Narrator seine erste optische Wahrnehmung dar: „Über die Mole kollerten verschimmelte Orangen aus der Ladung der Trivia Erinnerungen an die Gärten Italiens.“" ${ }^{80}$ Die Erinnerung weicht hier vom Urbild ab: Der verwandelte Zustand der Früchte zeigt die verflossene Zeit an. Die transformierende Erinnerung prägt also die Wahrnehmung in der Erzählgegenwart, deren zeitliche Kontinuität dennoch Leerstelle bleibt. Rückblenden auf vorheriges Geschehen beschränken sich nicht auf narrative Rekonstruktionen einer Erinnerung an Vergangenes. Die vielfältigen Erinnerungen in diesem Roman, der einen Protagonisten 
auf der Suche nach Erinnerungen zeigt, manifestieren sich in ebenso vielfältigen Formen: Sie werden personalisiert, versteinert, erzählt, verwandelt.

Die Chronotopoi des Romans heben die vermeintlichen Konstanten der Raum-ZeitKorrelation auf. ${ }^{81}$ Der diegetische Raum wird von zeitlichen Entwicklungen überlagert. Die Natur verändert sich, Geschwindigkeiten tierischer, pflanzlicher und geologischer Entwicklung variieren, neue Berge, Landschaften entstehen. ${ }^{82}$ Cotta indes wird die Abweichung von der topographischen Gewohnheit erst sukzessive klar. Schließlich erkennt er, ,daß sein Ort weder in der eisernen noch in der ewigen Stadt lag, sondern daß er in eine Zwischenwelt geraten war, in der die Gesetze der Logik keine Gültigkeit mehr zu haben schienen, [...]." ${ }^{\text {83 }}$ Cotta ist in eine Welt geraten, in der selbst Kontinuität und Chronologie der Zeit den Gesetzen der erzählten Erinnerung unterliegen, eine Welt, in der alles und alle veränderbar, transformierbar, verwandelbar erscheinen, eine Welt der Fiktion.

Nur ein Element des Romans unterliegt nicht diesen Metamorphosen: das Nashorn, das Augustus in seinen Gärten hält und „an dessen gewaltigem Körper der Lauf der Zeit keine Spuren zu hinterlassen schien; allein die Generationen von Fliegen, Ungeziefer und Vögeln, die das Nashorn auf seinem gepanzerten Rücken trug, alterten, starben und erneuerten sich, aber das Tier im Pfuhl, das alle diese Wesen ernährte und sie erdrückte, wenn es sich im Staub und im Schlamm wälzte, blieb sich in den Jahren gleich wie Stein. “" ${ }^{84}$ Das Nashorn verkörpert das Gegenmodell zur mythischen Metamorphose, das Überdauern, die Identität mit sich selbst. Es bedarf keiner Erinnerung und keiner Erneuerung, es bedarf keines Namens, es bleibt stets dasselbe, in seinem festgelegten, für ihn hergerichteten Raum, wo es „wild und lebendig ${ }^{\text {c }}{ }^{85}$ und doch "gleich wie ein Stein“ erscheinen kann. Das Beständige, der überdauernde Kern jedes Mythos steht indes in Kontakt mit den flüchtigen Aggregatzuständen der Verwandlung, mit dem aus Luft und Erde zusammengesetzten Staub und dem aus Wasser und Erde zusammengesetzten Schlamm. Der Kern des Mythos, das Überdauernde, bleibt aber letztlich unnennbar und unerklärbar.

III.

In den Erinnerungen der Figuren konstituiert sich die „letzte Welt“. Doch die erzählte Erinnerung an Vergangenes umgeht die Gegenwart, indem sie sie in einer „letzten“ Zukunft spiegelt. Der Zusammenhang von Text und Poetik besteht bei Ransmayr im Zusammenspiel von Erinnerung und Aus-der-Zeit-Sein. ${ }^{86}$ Ransmayrs Texte rücken aus der Zeit, nicht, indem sie alternative Entwürfe eines „was wäre, wenn?“ präsentieren, sondern indem sie den memorativ-imaginierten Charakter von Mythen neu schreiben. Die Verbindung von Text-Erinnerung und imaginativer Konstruktion lässt das tertium comparationis, die Gegenwart, aus. ${ }^{87}$ Das Miteinander von erzählter, individueller Erinnerung und Zukunft auszufüllen ist die Aufgabe des Rezipienten.

Der Mythos muss die Gegenwart der Figuren wie der Rezipienten nicht mehr erklären. Sie haben den ,willkürlichen' Glauben an die Wahrheit der Mythen verloren. Das mythische Aus-der-Zeit-Sein wird bei Ransmayr durch Erinnerung konstruiert, die sich im narrativen Akt nicht nur außerhalb konstanter Topographie, sondern auch außerhalb der zeitlichen Kontinuität situiert. Die literarische Repräsentation von Erinnerung erfordert und überschreitet den außer-zeitlichen Charakter von Ransmayrs Texten. Während Ovids Metamorphosen im letzten Buch eine für die Lesergegenwart 
gültige Erklärung für den Mythos liefern, wird bei Ransmayr der logisch-rationale Ordnungsraum vollständig aufgelöst. Erklärungen für die Gegenwart fehlen bei Ransmayr. Nur die negativen Folgen der Aufklärung (in Rom) und den indifferenten Mythos (in Tomi) zu kontrastieren und den Roman als Repräsentation der Dialektik der Aufklärung zu verstehen, würde aber die poetische Struktur von Ransmayrs Text um Wesentliches verkürzen ${ }^{88}$ Der Roman variiert die Grautöne der Arbeit am Mythos. In ihnen ist keine Eindeutigkeit erkennbar. Auch der Narrenzug in Tomi, von dem Ransmayr erzählt, ist nur ein (schwaches) Abbild, ein „stumpfer Abglanz“ der längst vergangenen Mythen Roms. ${ }^{89}$ Die vermeintlich mythischen Gestalten tragen nur deren Namen. Der subversive Effekt des Karnevals kann hier nicht als Emanzipation gelesen werden, denn der Narrenzug ist ein Pastiche, eine Nachahmung ohne kritischsatirische Absicht - ein Ort mehr, an dem Mytheme erscheinen und verändert werden.

Doch provoziert auch diese Form der Literatur eine Rezeptionshaltung, die sich nicht in Unbestimmtheiten verliert. Sie mobilisiert - so könnte man Theodor W. Adorno folgend formulieren - eine Wahrnehmung, durch die sich das Selbst zu einem anderen in Beziehung setzen kann. ${ }^{90}$ Nicht selten hat die Forschung Ransmayrs Die letzte Welt in Bezug zur Frankfurter Schule gesetzt. Der Weg scheint mir nicht falsch, doch vielleicht ist es erwägenswert, den vermeintlich offensichtlichen Zusammenhang des Romans zur Dialektik der Aufklärung durch die Bezüge zu Adornos Kunsttheorie zu ergänzen. Die Letzte Welt repräsentierte dann nicht das dialektische Ineinander von (falsch verstandener) Aufklärung und Mythos, sondern sie führte die Möglichkeiten der Kunst vor, indem sie den Rezipienten zu selbsttätiger Entzifferung von Uneindeutigkeiten ermächtigt, die die literarkritische Angst vor der Kontingenz wie die aufklärerische „Furcht vor der Wahrheit"“91 erträgt. Man verkürzte demnach Ransmayrs Roman und seine qua literarischer Form mögliche Funktion, wenn man ihn bloß als Repräsentation des dialektischen Verhältnisses von Aufklärung und Mythos verstünde..$^{92}$ Kunst ist nicht Abbild, sondern Spielbild. Sie repräsentiert nicht die Dialektik der Aufklärung (weder im mimetischen noch im nicht-mimetischen Sinne von „Repräsentieren“), sondern sie ermöglicht, sie zu reflektieren. ${ }^{93}$ Aber Rezeption kann nicht außerhalb des Prozesses stattfinden, auf den Mythen und Erinnerungen einwirken.

Nicht zufällig gerät Pythagoras, der in Ovids Metamorphosen die abschließende, gleichsam aufgeklärte Welt- und Mythoserklärung liefert, ${ }^{94}$ in Ransmayrs Text in den Verdacht, wahnsinnig zu sein. Die örtliche Distanz scheint auch eine geistige zu signalisieren: „In das Reich dieses Alten schien kein Weg zu führen.“" ${ }^{\text {" }}$ Doch sein Erzählen befreit Pythagoras aus der Isolation, indem es Fiktion (in der Fiktion) erzeugt. Und doch kann es nicht außerhalb des Systems stehen. Pythagoras ist Teil der erzählten und erzählenden Welt. Für den Leser konstituiert sich in den Erzählungen der Letzten Welt keine simple Sinnstiftung oder Identitätskonstruktion. Das Erzählen Ransmayrs könnte in diesem Sinne, Blumenberg folgend, mythisch genannt werden: Es sind Geschichten, denen nicht von der Wirklichkeit widersprochen werden kann. ${ }^{96}$ Ransmayrs Schreiben macht die Bedingungen der Möglichkeit des Erinnerns reflexionsfähig. Rezipienten werden dazu angehalten, über Möglichkeiten und Reichweite des Erinnerns nachzudenken. Die Abweichung von der gewohnten Rezeptionshaltung, die Leerstelle der Gegenwart, die „Deautomatisierung der Leserreaktion ${ }^{\text {“97 }}$ erzeugen hier rezeptive Aufmerksamkeit.

Der narrative Kern des Mythos wird in Ransmayrs Letzter Welt somit nicht nur variiert, sondern auch in einem neuen Ausdruck der „Formbestimmtheit" ${ }^{“ 98}$ transformiert. Die 
Mythen in Ransmayrs Roman unterscheiden sich von den antiken, indem sie das funktionelle Prinzip jedes Mythos radikalisieren: Ransmayrs neue Mythenform überantwortet durch die Neuverschränkung der Mytheme jedem Individuum die selbstbestimmte Möglichkeit, seine erzählte Erinnerung an die Welt und damit sein Bewusstsein von dieser $\mathrm{zu}$ reflektieren. Dass Bruchstellen, Elemente der Uneindeutigkeit und der Differenz sichtbar werden, ist funktional für den Prozess der Rezeption. ${ }^{99}$ Sie werden zum Teil des Mythos. Die von Ransmayr provozierte „bricolage“ 100 führt indes nicht zur mythologischen Welterklärung wie im traditionellen mythologischen Denken, sondern zur Reflexion des Zusammenhangs, wie Mythen durch erinnerte Erzählung produziert, tradiert oder destruiert werden. Ransmayrs Text erlaubt es, die Funktionsgesetze von Mythen metaphorisch zu verstehen und in Fiktion zu übertragen. ${ }^{101}$ Ransmayr verwendet den Mythos zur funktionalen Erklärung einer Poetologie der Erinnerung. Erzählen bildet weder reale Vergangenheit noch das mythische Erbe ab, es schafft sie neu, es formt sie zur individuellen Erinnerung um, es eignet sie sich an. Das ist nach Ransmayr „die Verzauberung des Lebens in Lieder“. ${ }^{102}$

\section{NOTES}

1. Vgl. u.a. David Malouf: An Imaginary Life (1978), Salman Rushdie: The Satanic Verses (1988), Viktor Pelewin: Helmet of Horror (2005).

2. Vgl. Thomas Anz: Spiel mit der Überlieferung. Aspekte der Postmoderne in Ransmayrs Die letzte Welt; in: Die Erfindung der Welt. Zum Werk von Christoph Ransmayr. Hrsg. von Uwe Wittstock, Frankfurt/M. 1997, S. 120-132, hier S. 121f. Anz bezieht sich auf Jacques Derrida: Die Struktur, das Zeichen und das Spiel im Diskurs der Wissenschaften vom Menschen; in: ders.: Die Schrift und die Differenz. Übs. von Rodolphe Gasché, Frankfurt/M. 1976, S. 422-442, v.a. S. 428ff., Claude LéviStrauss: Strukturale Anthropologie I. Übs. von Hans Naumann, Frankfurt/M. 1977, S. $226 f f$.

3. Vgl. Anz: Spiel, S. 121.

4. Anz bezieht sich auf Hans Blumenberg: Arbeit am Mythos, Frankfurt/M. ${ }^{5} 1990$.

5. Christian Schärf: Der Roman im 20. Jahrhundert, Stuttgart, Weimar 2001, S. 176.

6. Vgl. Kurt Bartsch: Dialog mit Antike und Mythos. Christoph Ransmayrs Ovid-Roman „Die letzte Welt"; in: Modern Austrian Literature 23 (1990). N. 3/4, S. 121-133.

7. Vgl. Karlheinz Töchterle: Spiel und Ernst - Ernst und Spiel. Ovid und „Die letzte Welt“ von Christoph Ransmayr; in: Antike und Abendland 28 (1992), S. 95-106, Reinhold F. Glei: Ovid in den Zeiten der Postmoderne. Bemerkungen zu Christoph Ransmayrs Roman ,Die letzte Welt'; in: Poetica 26 (1994), S. 409-427. Positiv hebt sich die gründliche Arbeit von Barbara Vollstedt ab, die Übereinstimmungen und Abweichungen zwischen Ovid-Texten und Die letzte Welt im Detail beschreibt. Vgl. Barbara Vollstedt: Ovids „Metamorphoses“, „Tristia“ und „Epistulae ex Ponto“ in Christoph Ransmayrs Roman „Die letzte Welt“, München, Wien u.a. 1998.

8. Vgl. Peter Bachmann: Die Auferstehung des Mythos in der Postmoderne. Philosophische Voraussetzungen zu Christoph Ransmayrs Roman ,Die letzte Welt'; in: Diskussion Deutsch 21 (1990), S. 639-651, hier S. 650f.

9. Vgl. dagegen Harzers These, in Ransmayrs Roman zeige sich eine „neostrukturalistische Poetik epischer Metamorphosen“. Vgl. Friedemann Harzer: Erzählte Verwandlung. Eine Poetik epischer Metamorphosen (Ovid - Kafka - Ransmayr), Tübingen 2000, S. $175 f$. 
10. Christoph Ransmayr: Geständnisse eines Touristen. Ein Verhör, Frankfurt/M. ${ }^{2} 2004$, S. 116.

11. Christoph Ransmayr: Die dritte Luft oder Eine Bühne am Meer, Frankfurt/M. 1997, S. 11. Vgl. zur folgenden Interpretation von Ransmayrs Autorpoetik Verf.: Uchronische Erinnerung und erinnerte Uchronie. Zur Poetik Christoph Ransmayrs; in: Gegenwartsliteratur. Ein germanistisches Jahrbuch. A German Studies Yearbook 7 (2008), S. 182-203.

12. Christoph Ransmayr: Die Erfindung der Welt. Rede zur Verleihung des Franz-Kafka-Preises; in: Uwe Wittstock (Hrsg.): Die Erfindung der Welt. Zum Werk von Christoph Ransmayr, Frankfurt/M. 1997, S. 198-202, hier S. 200.

13. Ransmayr: Dritte Luft, S. 15.

14. Ebd., S. 16. Hervorhebungen R.G.

15. Ransmayr: Geständnisse, S. 14.

16. Blumenberg: Arbeit, S. 13.

17. Ransmayr: Dritte Luft, S. 19.

18. Vgl. Jean-François Lyotard: Das postmoderne Wissen. Ein Bericht. Übs. von Otto Pfersmann, hrsg. von Peter Engelmann, Graz, Wien 1986, S. 112.

19. Ransmayr: Dritte Luft, S. 20.

20. Ebd., S. 24.

21. Vgl. zum „New Historicism“ in Bezug auf Ransmayr Andrea Kunne: Heimat und Holocaust. Aspekte österreichischer Identität aus postmoderner Sicht. Christoph Ransmayrs Roman Morbus Kitahara; in: Amsterdamer Beiträge zur neueren Germanistik 49 (2000): Postmoderne Literatur in deutscher Sprache: Eine Ästhetik des Widerstands? Hrsg. von Henk Harbers, S. 311-333.

22. Vgl. Blumenberg: Arbeit, S. 177.

23. Vgl. Lévi-Strauss: Strukturale Anthropologie, S. $229 f$.

24. Vgl. Blumenberg: Arbeit, S. 300.

25. Vgl. ebd., S. 302.

26. Vgl. Stephanie Wodianka: Mythos und Erinnerung. Mythentheoretische Modelle und ihre gedächtnistheoretischen Implikationen; in: Günter Oesterle (Hrsg.): Erinnerung, Gedächtnis, Wissen. Studien zur kulturwissenschaftlichen Gedächtnisforschung, Göttingen 2005, S. 211-230, hier S. 215.

27. Hans Blumenberg: Wirklichkeitsbegriff und Wirkungspotential des Mythos; in: Manfred Fuhrmann (Hrsg.): Terror und Spiel. Probleme der Mythenrezeption, München 1971, S. 11-66, hier S. $49 f$.

28. Vgl. Blumenberg: Arbeit, S. 28, 295.

29. Vgl. zur Vorgeschichte Vollstedt: Ovids „Metamorphoses“, S. 25ff., Angela Fitz: „Wir blicken in ein ersonnenes Sehen." Wirklichkeits- und Selbstkonstruktion in zeitgenössischen Romanen. Sten Nadolny, Christoph Ransmayr, Ulrich Woelk, St. Ingbert 1998, S. 215.

30. Erstmals in: Andreas Thalmayr (= Hans Magnus Enzensberger, Hrsg.): Das Wasserzeichen der Poesie oder die Kunst und das Vergnügen, Gedichte zu lesen: in hundertvierundsechzig Spielarten, Frankfurt/M. 1990. Hier zit. nach: Christoph Ransmayr: Das Labyrinth. Eine Baugeschichte aus Kreta; in: ders.: Der Weg nach Surabaya. Reportagen und kleine Prosa, Frankfurt/M. 1999, S. 211-214.

31. P. Ovidius Naso: Metamorphosen. Lateinisch / Deutsch. Übs. und hrsg. von Michael von Albrecht, Stuttgart 2003, VIII 167f.

32. Ransmayr: Labyrinth, S. 214.

33. Ransmayr publizierte im Anschluss seinen Entwurf $z u$ einem Roman, der hier nicht ausführlicher analysiert werden kann. Vgl. Christoph Ransmayr: Entwurf zu einem Roman; in: Jahresring. Jahrbuch für Kunst und Literatur 34 (1987/88), S. 196-198. Vgl. hierzu Thorsten Wilhelmy: Legitimitätsstrategien der Mythosrezeption. Thomas Mann, Christa Wolf, John Barth, Christoph Ransmayr, John Banville, Würzburg 2004, S. 283f., Anz: Spiel.

34. Vgl. Blumenberg: Arbeit, S. 165.

35. Vgl. Wodianka: Mythos, S. $212 \mathrm{f}$. 
36. Und darüber hinaus, wie Vollstedt nachweist, auf weitere Ovid-Texte. Vgl. Vollstedt: Ovids "Metamorphoses".

37. Ovid: Metamorphosen I $5 \mathrm{ff}$.

38. Vgl. Blumenberg: Arbeit, S. 384.

39. Vgl. Roland Barthes: Mythen des Alltags. Dt. von Helmut Scheffel, Frankfurt/M. 1964, S. 110ff. Nicht geteilt werden hier die Überlegungen von Barthes zur grundständigen Differenz von Mythos und Poesie. Vgl. ebd., S. 117f.

40. Ransmayr: Letzte Welt, S. 198; vgl. Monika Schmitz-Emans: Christoph Ransmayr: Die Letzte Welt (1988) als metaliterarischer Roman; in: Anselm Maler, Ángel San Miguel, Richard Schwaderer (Hrsg.): Europäische Romane der Postmoderne, Frankfurt/M. 2004, S. 119-148, hier S. 128, Monica Fröhlich: Literarische Strategien der Entsubjektivierung. Das Verschwinden des Subjekts als Provokation des Lesers in Christoph Ransmayrs Erzählwerk, Würzburg 2001, S. 92.

41. Ovid: Metamorphosen XV 876.

42. Vgl. Ransmayr: Die letzte Welt, S. $49 \mathrm{ff}$.

43. Ebd., S. 51.

44. Ovid: Metamorphoses XV 877.

45. Vgl. ebd. VII 501ff., Vollstedt: Ovids „Metamorphosen“, S. 35.

46. Ovid: Metamorphosen VII $655 f$.

47. Ransmayr: Letzte Welt, S. 64.

48. Vgl. Vollstedt: Ovids „Metamorphosen“, S. 38.

49. Indes gelingt die Ansprache des Publikums nicht, das offenbar die Dialektik von „beherrschbar" und „unbesiegbar" auf das Letztere reduziert und die Zukunftsoption für eine Beschreibung der Gegenwart hält. Der Apparat aber nimmt die Ansprache Nasos zum Anlass, seine Verbannung aus Rom einzuleiten. Vgl. Ransmayr: Letzte Welt, S. 64ff., $66 \mathrm{ff}$.

50. Ovid hat hier das bereits traditionelle Motiv der "Deucalionis aquae“ aufgenommen. Vgl. auch zu Unterschieden in Handlungsmotivation und -erzählung Vollstedt: Ovids „Metamorphosen “, S. $66 \mathrm{ff}$.

51. Ovid: Metamorphosen I 414f. Hervorhebungen R.G.

52. Vgl. die Prophezeiung Jupiters ebd. I 251f.

53. Ransmayr: Letzte Welt, S. 169.

54. Ebd., S. 162.

55. Ebd., S. 161.

56. Ebd., S. 162f. Vgl. Vollstedt: Ovids „Metamorphosen“, S. $73 f$.

57. Ransmayr: Letzte Welt, S. 169.

58. Das „Ende der Welt“ ist Figurenperspektive. Vgl. Ransmayr: Die letzte Welt, S. 188. Ob eine deszendente und pessimistische Kulturtheorie Ransmayr zuzuschreiben ist, muss fraglich bleiben. Vgl. Vollstedt: Ovids „Metamorphosen“, S. 79, Harzer: Erzählte Verwandlung, S. 179ff. Schon gar nicht kann eine Erwartung der Apokalypse dem Autor Ransmayr zugerechnet werden. Vgl. Thomas Epple: Christoph Ransmayr. Die letzte Welt. Interpretation, München 1992, S. 54f. Vgl. zur Kritik an Epple bereits begründet Fitz: Ersonnenes Sehen, S. 222.

59. Ransmayr: Letzte Welt, S. 162.

60. Vgl. Wilhelmy: Legitimitätsstrategien, S. 303.

61. Vgl. zur Erzählstrategie dieser Passagen Fröhlich: Literarische Strategien, S. 94, zur Emotionalität der Situation Fitz: Ersonnenes Sehen, S. 232.

62. Vgl. zum Uchronie-Konzept ausführlicher Verf.: Uchronische Erinnerung.

63. Ernst Cassirer: Philosophie der symbolischen Formen. Zweiter Teil. Das mythische Denken. Text und Anmerkungen bearb. von Claus Rosenkranz, Hamburg 2002 (= Gesammelte Werke. Hamburger Ausgabe. Hrsg. von Birgit Recki. Bd. 12), S. 18.

64. Vgl. Ernst Cassirer: The Myth of the State, New York ${ }^{2} 1955$, S. 57f., auch Blumenberg: Wirklichkeitsbegriff, S. 14. 
65. Das Repertoire nur als Aufforderung zu lesen, Beziehungen zwischen Ovid und Ransmayr zu entdecken (Vollstedt: Ovids „Metamorphosen“, S. 13), greift ebenso zu kurz wie dessen Abwertung (vgl. Herwig Gottwald: Mythos und Mythisches in der Gegenwartsliteratur. Studien zu Christoph Ransmayr, Peter Handke, Botho Strauß, George Steiner, Patrick Roth und Robert Schneider, Stuttgart 1996).

66. Vgl. Blumenberg: Arbeit, S. 40.

67. Vollstedt bemerkt zurecht, dass die Überschrift „Ein Ovidisches Repertoire“ lautet, dass es sich also nur um eine der möglichen Formen handelt. Vgl. Vollstedt: Ovids „Metamorphosen“, S. 187.

68. Vgl. Duncan F. Kennedy: Recent receptions of Ovid; in: The Cambridge Companion to Ovid. Ed. by Philip Hardie, Cambridge 2002, S. 320-335, hier S. 326.

69. Ransmayr: Dritte Luft, S. 15.

70. Ebd., S. 16.

71. Ebd., S. 26.

72. Vgl. Vollstedt: Ovids „Metamorphosen“, S. 187.

73. Vgl. Jaroslav Kovář: Acht Thesen zu Christoph Ransmayrs Roman ,Die letzte Welt; in: Literatur und Kritik 25 (1990), S. 193-200, hier S. 196f., Ralf Peter Märten: Ransmayrs Rom. Der Poet als Historiker; in: Die Erfindung der Welt. Zum Werk von Christoph Ransmayr. Hrsg. von Uwe Wittstock, Frankfurt/M. 1997, S. 113-119, hier S. 113f. Märten erkennt, dass der Verzicht auf historische Stimmigkeit für das Funktionieren des Romans relevant ist. Vgl. ebd., S. 118, daneben auch Schmitz-Emans: Ransmayr, S. 122, Epple: Ransmayr, S. 12.

74. Vgl. Wilhelmy: Legitimitätsstrategien, S. 279, 310.

75. Ransmayr: Geständnisse, S. 93.

76. Ransmayr: Letzte Welt, S. 9.

77. Ebd., S. 8.

78. Ebd., S. 9.

79. Ebd., S. 241.

80. Ebd., S. 8.

81. „Chronotopos“ verstehe ich dabei mit Bachtin als erzähltheoretische „Form-InhaltKategorie“ der Literatur. Vgl. Michail M. Bachtin: Chronotopos. Aus dem Russ. von Michael Dewey. Mit einem Nachwort von Michael C. Frank und Kirsten Mahlke, Frankfurt/M. 2008, S. 7. Nicht intendiert ist eine andere Lesart, die Bachtin ebenfalls nahelegt: den Chronotopos als kulturtheoretische Kategorie zu verstehen. Vgl. ebd., S. 186f., Gary Saul Morson, Caryl Emerson: Mikhail Bakhtin. Creation of a Prosaics, Stanford / CA 1990, S. $295 \mathrm{ff}$.

82. Vgl. Ransmayr: Letzte Welt, S. $284 \mathrm{f}$.

83. Ebd., S. 220.

84. Ebd., S. $128 f$.

85. Ebd., S. 94.

86. Vgl. hierzu Verf.: Uchronische Erinnerung.

87. Erinnerung wird in neuerer Forschung nicht selten als Konzept bestimmt, Vergangenheit und Zukunft in der Gegenwart aufeinander zu beziehen. Vgl. Günter Oesterle: Einleitung; in: ders. (Hrsg.): Erinnerung, Gedächtnis, Wissen. Studien zur kulturwissenschaftlichen Gedächtnisforschung, Göttingen 2005, S. 11-23, hier S. 13.

88. Vgl. u.a. Sabine Wilke: Poetische Strukturen der Moderne. Zeitgenössische Literatur zwischen alter und neuer Mythologie, Stuttgart 1992, S. 37, Epple: Ransmayr, S. 94.

89. Ransmayr: Letzte Welt, S. 93.

90. Vgl. Theodor W. Adorno: Ästhetische Theorie. Hrsg. von Gretel Adorno und Rolf Tiedemann, Frankfurt/M. ${ }^{13} 1995$, S. 24ff. (in Kritik an Kant und Freud) et passim.

91. Max Horkheimer, Theodor W. Adorno: Dialektik der Aufklärung. Philosophische Fragmente, Frankfurt/M. 1988, S. 4. 
92. Vgl. in diesem Sinne Wilke: Poetische Strukturen, S. 244.

93. Vgl. Kennedy: Recent receptions, S. 327.

94. Vgl. Ovid: Metamorphosen XV 60ff. Pythagoras kann allerdings auch bei Ovid nicht als „Rationalist“ verstanden werden. In dieser Hinsicht wäre Wilhelmy: Legitimitätsstrategien, S. 287 zu korrigieren.

95. Ransmayr: Letzte Welt, S. 17.

96. Vgl. Blumenberg: Arbeit, S. 13.

97. Vgl. Markus Oliver Spitz: Erfundene Welten - Modelle der Wirklichkeit. Zum Werk von Christoph Ransmayr, Würzburg 2004, S. 166.

98. Cassirer: Philosophie, S. 16.

99. Vgl. Wilhelmy: Legitimitätsstrategien, S. 319f.

100. Vgl. Lévi-Strauss: Strukturale Anthropologie, S. 231.

101. Vgl. Wilhelmy: Legitimitätsstrategien, S. 306, Harzer: Erzählte Verwandlung, S. 184, Bachmann: Auferstehung, S. 649.

102. Ransmayr: Dritte Luft, S. 26.

\section{RÉSUMÉS}

Der Artikel untersucht die Verarbeitung antiker Mythen in Christoph Ransmayrs Roman Die letzte Welt (1988). Ransmayr macht hier Ovids Verwandlungsmythen zum Material eines Spiels, das die Metamorphosen weiter- und umerzählt. Unter Rückgriff auf Ransmayrs eigene Poetik der Erinnerung wird analysiert, welche Funktion die Transformation des Mythos im literarischen Wirkungszusammenhang bei Ransmayr übernimmt. Dabei wird eine poetologisch unterlegte Differenz deutlich: Ransmayrs Roman zielt darauf, mit seiner literarischen Umformung des Mythos Geschichten unter den Bedingungen der Gegenwart neu zu erzählen. Diese Geschichten offenbaren Möglichkeiten, die sich in der Vorstellungskraft des Erzählers und der Rezipienten konkretisieren sollen. Der Roman führt damit vor, wie Erzählen als Form der Erinnerung gleich den Mythen Vergangenes gestaltet. Die letzte Welt arbeitet also dadurch am Mythos (im Sinne Blumenbergs), dass sie den Textbestand von Ovid variiert und überschreitet, aber die Funktion des Mythos für die Gegenwart adaptiert, den Rezipienten zum narrativen Umgang mit individueller Erinnerung zu befähigen.

This article analyzes the adaption of antique myths in Christoph Ransmayr's novel Die letzte Welt (1988). Ransmayr "playfully" re-narrates the transformative myths from Ovid's Metamorphoses. Drawing upon Ransmayr's own poetics of memory, this article explores the question of what function the transformation of myths fulfills within Ransmayr's literary context. In so doing, a poetological difference becomes clear: Ransmayr's novel aims at a new narration of mythological stories in the context of the present day. These stories offer new possibilities that are realised in the imagination of the narrator and the reader. On this way, the novel demonstrates how narration as a form of memory models the past - just as myths do. Die letzte Welt "works" at the myth (in the sense of Blumenberg) by modifying and going beyond Ovid's text, but it also adapts its function for the present, by enabling the reader to deal with his individual memories in a narrative way. 
Cet article porte sur la façon dont Christoph Ransmayr a retravaillé les mythes antiques dans le roman de Christoph Ransmayr Die letzte Welt (1988). En se fondant sur le mythe des métamorphoses tel qu'il existe chez Ovide, l'auteur du roman en poursuit et modifie, de manière ludique, la narration. Le recours à la poétique du souvenir caractéristique de Ramsmayr permet d'analyser la fonction jouée par la transformation du mythe dans un contexte littéraire. Cette analyse met en lumière la spécificité poétologique de l'écriture de Ramsmayr: par la transformation littéraire du mythe, il s'efforce d'en proposer une narration nouvelle, dans les conditions propres à l'époque actuelle. Les histoires qu'il narre offrent un certain nombre de possibilités, qui se réalisent dans l'imagination du narrateur et du lecteur. Par là, le roman montre bien comment la narration, en tant que forme du souvenir, modèle le passé, ainsi que le font les mythes. Dans Die letzte Welt, le mythe a été retravaillé (dans le sens que prête à ce terme Blumenberg) en ce que le texte a été modifié et dépasse le texte que nous a laissé Ovide, tout en modifiant, afin de l'adapter aux conditions présentes, la fonction du mythe, fonction qui consiste à rendre le lecteur capable de jouer, de manière narrative, avec le souvenir individuel.

\section{INDEX}

oeuvrecitee Die letzte Welt, Métamorphoses

\section{AUTEURS}

\section{RAINER GODEL}

Universität Halle/Saale 